\title{
A Compensated Numerical Method for Solving Stochastic Differential Equations with Variable Delays and Random Jump Magnitudes
}

\author{
Ying Du and Changlin Mei \\ Department of Statistics, School of Mathematics and Statistics, Xian Jiaotong University, Xian 710049, China \\ Correspondence should be addressed to Ying Du; duyingxjtu@aliyun.com
}

Received 29 September 2014; Revised 13 January 2015; Accepted 14 January 2015

Academic Editor: Chin-Chia Wu

Copyright (c) 2015 Y. Du and C. Mei. This is an open access article distributed under the Creative Commons Attribution License, which permits unrestricted use, distribution, and reproduction in any medium, provided the original work is properly cited.

Stochastic differential equations with jumps are of a wide application area especially in mathematical finance. In general, it is hard to obtain their analytical solutions and the construction of some numerical solutions with good performance is therefore an important task in practice. In this study, a compensated split-step $\theta$ method is proposed to numerically solve the stochastic differential equations with variable delays and random jump magnitudes. It is proved that the numerical solutions converge to the analytical solutions in mean-square with the approximate rate of $1 / 2$. Furthermore, the mean-square stability of the exact solutions and the numerical solutions are investigated via a linear test equation and the results show that the proposed numerical method shares both the mean-square stability and the so-called A-stability.

\section{Introduction}

Stochastic differential equations (SDEs) for jump-diffusions arise in a variety of practical areas and have successfully been used to describe unexpected and abrupt changes in the present structure (for an overview, see $[1,2]$ ). Generally, SDEs for jump-diffusions cannot be solved explicitly. Therefore, constructing some forms of numerical solution and studying their properties have received a great deal of attention. When the jump magnitude is deterministic, Higham and Kloeden [3-5] studied the convergence and stability of the numerical solutions of the stochastic differential equations with jumps. Wang et al. [6] proved that the semi-implicit numerical solutions of stochastic delay differential equations with jumps are convergent to their corresponding analytical solutions. Jiang et al. [7] investigated the Taylor approximation for stochastic delay differential equation with jumps (SDDEJs). Bao et al. [8] obtained the convergence rate of the EulerMaruyama method for SDDEJs under the local Lipschitz condition. Furthermore, some researchers [9-11] extended the constant delay $\tau$ in SDDEJs to variable delay $\tau(t)$.

Chalmers and Higham [12] extended the results in [4] to the case where the jump magnitudes are random. Stochastic differential equations with compound Poisson processes have been commonly used in mathematical finance and covering a wide range of finance models [13-16]. Considering the aftereffect of the past state, Jiang et al. [17] proposed a semiimplicit Euler numerical method for stochastic differential delay equations with Poisson driven jumps of random magnitudes. Furthermore, Mao [18] studied the stochastic differential equations with variable delays and random jump magnitudes (SDEVDRJMs) which are of the form

$$
\begin{aligned}
\mathrm{d} x(t)= & f(x(t), x(t-\tau(t))) \mathrm{d} t \\
& +g(x(t), x(t-\tau(t))) \mathrm{d} W(t) \\
& +h\left(x(t), x(t-\tau(t)), \gamma_{N(t)+1}\right) \mathrm{d} N(t), \\
& t \in[0, T] ; \\
x(t)= & \xi(t), \quad t \in[-r, 0],
\end{aligned}
$$

where $\tau(t)$ is a variable delay, $W(t)$ is an $m$-dimensional standard Wiener process, $N(t)$ is a scalar Poisson process with intensity $\lambda, \gamma_{i}(i=1,2, \ldots)$ are independent and identically distributed random variables representing jump magnitudes, 
the drift coefficient $f(x, y)$ and the jump coefficient $h(x, y, \gamma)$ are $\mathbb{R}^{n}$-valued, the diffusion coefficient $g(x, y)$ is $\mathbb{R}^{n \times m}$ valued for $x, y \in \mathbb{R}^{n}$, and $\xi(t)$ is starting delay condition function. Moreover, it is generally assumed that, for some $p \geq 2$, there exists a constant $B$ such that $\mathbb{E}\left[\left|\gamma_{i}\right|^{p}\right] \leq B$. Mao [18] proposed a semi-implicit Euler numerical method for SDEVDRJMs and proved that the numerical solutions converge to their analytical solution both in mean-square and in probability.

However, as pointed out by Chalmers and Higham [12], the semi-implicit Euler method for stochastic differential equations with random jump magnitudes is stable in meansquare but lose the A-stable result for constant jump magnitude. Here, A-stable property means that "problem stable $\Rightarrow$ method stable for all time step size $\Delta$." Furthermore, it seems that there is little literature on the study of the stability of numerical methods for SDEVDRJMs.

Our work is motivated by [3], where compensated splitstep backward Euler method is introduced and proved to have a satisfactory stability property. In this paper, a compensated split-step $\theta$ method is proposed to construct the numerical solutions of SDEVDRJMs, and the mean-square convergence of the numerical solutions is proved. Furthermore, the meansquare stability of the analytical solutions and the numerical solutions is investigated via a test equation; the results demonstrate that the proposed numerical method possesses not only the mean-square stability but also the A-stability.

\section{Compensated Split-Step $\theta$ Numerical Solutions for SDEVDRJMs}

Let $\left(\Omega, \mathscr{F},\left\{\mathscr{F}_{t}\right\}_{t \in[0, T]}, \mathbb{P}\right)$ be a complete probability space with the filtration $\left\{\mathscr{F}_{t}\right\}_{t \in[0, T]}$ satisfying the usual conditions that $\mathscr{F}_{t}$ is right-continuous and $\mathscr{F}_{0}$ contains all $\mathbb{P}$-null sets. Let $|\cdot|$ be the Euclidean norm in $\mathbb{R}^{n}$ and let $\langle\cdot\rangle$ be the scalar product of vectors $x, y \in \mathbb{R}^{n}$. Denote $\|z\|_{L_{2}}=\left(\mathbb{E}\left[|z|^{2}\right]\right)^{1 / 2}$ with $z$ being a $n$-dimensional real random variable.

We firstly introduce the following assumptions for the establishment of the convergence and stability of the proposed numerical solution of the SDEVDRJMs in (1).

(i) The time delay $\tau:[0, \infty] \rightarrow \mathbb{R}$ satisfies

$$
0 \leq \tau(t) \leq t+r
$$

For $t, s \geq 0$, there exists positive constant $K_{1}$ such that

$$
\mathbb{E}(|\tau(t)-\tau(s)|) \leq K_{1}|t-s|
$$

(ii) Global Lipschitz condition: there exists a positive constant $K_{2}$ such that, for any $x_{k}, y_{k}, z_{k} \in \mathbb{R}^{n}(k=1,2)$, letting $y(t)=x(t-\tau(t))$,

$$
\begin{aligned}
& \left|f\left(x_{1}, y_{1}\right)-f\left(x_{2}, y_{2}\right)\right|^{2}+\left|g\left(x_{1}, y_{1}\right)-g\left(x_{2}, y_{2}\right)\right|^{2} \\
& \leq K_{2}\left(\left|x_{1}-x_{2}\right|^{2}+\left|y_{1}-y_{2}\right|^{2}\right) \\
& \left|h\left(x_{1}, y_{1}, z_{1}\right)-h\left(x_{2}, y_{2}, z_{2}\right)\right|^{2} \\
& \leq K_{2}\left(\left|x_{1}-x_{2}\right|^{2}+\left|y_{1}-y_{2}\right|^{2}+\left|z_{1}-z_{2}\right|^{2}\right) .
\end{aligned}
$$

(iii) Quadratic growth condition: for any $x_{k} \in \mathbb{R}^{n}(k=$ $1,2,3)$, there exists a positive constant $K_{3}$ such that

$$
\begin{aligned}
& \left|f\left(x_{1}, x_{2}\right)\right|^{2}+\left|g\left(x_{1}, x_{2}\right)\right|^{2} \leq K_{3}\left(1+\left|x_{1}\right|^{2}+\left|x_{2}\right|^{2}\right), \\
& \left|h\left(x_{1}, x_{2}, x_{3}\right)\right|^{2} \leq K_{3}\left(1+\left|x_{1}\right|^{2}+\left|x_{2}\right|^{2}+\left|x_{3}\right|^{2}\right) .
\end{aligned}
$$

(iv) For $-r \leq t, s \leq 0$, there exist positive constants $K_{4}$ and $K_{5}$ such that $\mathbb{E}|\xi(t)-\xi(s)| \leq K_{4}|t-s|$ and $\sup _{-r \leq t \leq 0}|\xi(t)|^{2} \leq K_{5}$.

Theorem 1. Under the assumptions (i) and (iii), there exist positive constants $C_{1}$ and $C_{2}$, such that the solution of the SDEVDRJM in (1) satisfies

$$
\begin{aligned}
& \mathbb{E}\left(\sup _{-r \leq t \leq T}|x(t)|^{2}\right) \leq C_{1}, \\
& \mathbb{E}\left(|x(t)-x(s)|^{2}\right) \leq C_{2}(t-s),
\end{aligned}
$$

for any $0 \leq s \leq t \leq T$.

Proof. From (1) and the linear growth condition, it can be obtained that, for any $t_{1} \in[0, T]$,

$$
\begin{aligned}
\mathbb{E}\left(\sup _{0 \leq t \leq t_{1}}|x(t)|^{2}\right) \\
\leq 4 \mathbb{E}\left(|\xi(0)|^{2}\right)+16 B K_{3}^{2} T+4\left(T+1+2 \lambda^{2} T+4 \lambda\right) \\
\cdot K_{3}^{2} \mathbb{E}\left(\sup _{0 \leq t \leq t_{1}} \int_{0}^{t}(1+|x(s)|+|x(s-\tau(s))|)^{2} \mathrm{~d} s\right) .
\end{aligned}
$$

Let $J_{1}=12\left(T+1+2 \lambda^{2} T+4 \lambda\right) K_{3}^{2}$ and $J_{2}=4 \sup _{-r \leq t \leq 0} \mathbb{E}|\xi(t)|^{2}+$ $16 B K_{3}^{2} T+J_{1}$. We have

$$
\begin{aligned}
& \mathbb{E}\left(\sup _{-r \leq t \leq t_{1}}|x(t)|^{2}\right) \\
& \quad \leq J_{2}+2 J_{1} \int_{0}^{t_{1}} \mathbb{E}\left(\sup _{-r \leq v \leq s}|x(v)|^{2}\right) \mathrm{d} s .
\end{aligned}
$$

Using Gronwall inequality, we obtain

$$
\mathbb{E}\left(\sup _{-r \leq t \leq T}|x(t)|^{2}\right) \leq J_{2} e^{2 J_{1} T}=C_{1} .
$$

Furthermore,

$$
\begin{aligned}
& \mathbb{E}\left(|x(t)-x(s)|^{2}\right) \\
& \leq 16 B K_{3}^{2}(t-s) \\
& +\frac{J_{1}}{3} \int_{s}^{t} \mathbb{E}\left[(1+|x(s)|+|x(s-\tau(s))|)^{2}\right] \mathrm{d} s \\
& \leq\left(16 B K_{3}^{2}+J_{1}+J_{1} C_{1}\right)(t-s) \leq C_{2}(t-s)
\end{aligned}
$$


Using the compensated Poisson process $\bar{N}(t)=N(t)-\lambda \Delta$ which is a martingale, we can rewrite

$$
\begin{aligned}
\mathrm{d} x(t)= & f_{\lambda}\left(x(t), x(t-\tau(t)), \gamma_{N(t)+1}\right) \mathrm{d} t \\
& +g(x(t), x(t-\tau(t))) \mathrm{d} W(t) \\
& +h\left(x(t), x(t-\tau(t)), \gamma_{N(t)+1}\right) \mathrm{d} \bar{N}(t),
\end{aligned}
$$

where

$$
\begin{aligned}
& f_{\lambda}\left(x(t), x(t-\tau(t)), \gamma_{N(t)+1}\right) \\
&= f(x(t), x(t-\tau(t))) \\
&+\lambda h\left(x(t), x(t-\tau(t)), \gamma_{N(t)+1}\right) .
\end{aligned}
$$

Obviously, $f_{\lambda}\left(x(t), x(t-\tau(t)), \gamma_{N(t)+1}\right)$ still satisfies the global Lipschitz condition and the linear growth condition. However, this equation is complicated and its analytical solution is in general hard to be obtained. In what follows, we use the compensated split-step $\theta$ method to construct its numerical solution.

For a given constant time step size $\Delta$, let

$$
\begin{aligned}
& m=\left[\frac{(n \Delta-\tau(n \Delta))}{\Delta}\right], \\
& \bar{m}=\left[\frac{((n+1) \Delta-\tau((n+1) \Delta))}{\Delta}\right] .
\end{aligned}
$$

Then the compensated split-step $\theta$ numerical solutions of the SDEVDRJM is of the form

$$
\begin{aligned}
x_{k}^{*}=x_{k}+[ & \theta f_{\lambda}\left(x_{k}^{*}, x_{\bar{m}}, \gamma_{N\left(t_{k}\right)+1}\right) \\
+ & \left.(1-\theta) f_{\lambda}\left(x_{k}, x_{m}, \gamma_{N\left(t_{k}\right)+1}\right)\right] \Delta ; \\
x_{k+1}= & x_{k}^{*}+g\left(x_{k}^{*}, x_{m}\right) \Delta W_{k} \\
& +h\left(x_{k}^{*}, x_{m}, \gamma_{N\left(t_{k}\right)+1}\right) \Delta \bar{N}_{k},
\end{aligned}
$$

where $x_{k}$ is the numerical approximation to $x\left(t_{k}\right), \Delta W_{k}=$ $W_{t_{k+1}}-W_{t_{k}}$, and $\Delta N_{k}=N_{t_{k+1}}-N_{t_{k}}$ for $t_{k}=k \Delta$ with $k=0$, $1, \ldots, N$ and $N=[T / \Delta]$ being the integer part of $T / \Delta$.

\section{Convergence of the Numerical Solutions}

In this section, we will prove that the above numerical solutions converge in mean-square to the true solution of the SDEVDRJM with the approximate rate of $1 / 2$.

Replacing the numerical approximations with the exact solution values on the right-hand side of (14a) and (14b), we obtain

$$
\begin{aligned}
\bar{x}_{k}^{*}=x\left(t_{k}\right)+\left[\theta f_{\lambda}\left(\bar{x}_{k}^{*}, x\left(t_{\bar{m}}\right), \gamma_{N\left(t_{k}\right)+1}\right)\right. & \left.+(1-\theta) f_{\lambda}\left(x\left(t_{k}\right), x\left(t_{m}\right), \gamma_{N\left(t_{k}\right)+1}\right)\right] \Delta \\
& +15 \\
\bar{x}_{k+1}= & \bar{x}_{k}^{*}+g\left(\bar{x}_{k}^{*}, x\left(t_{m}\right)\right) \Delta W_{k} \\
& +h\left(\bar{x}_{k}^{*}, x\left(t_{m}\right), \gamma_{N\left(t_{k}\right)+1}\right) \Delta \bar{N}_{k} .
\end{aligned}
$$

Then, the local error of the compensated split-step $\theta$ method for the approximation of the solution $x(t)$ of (1) is

$$
\delta_{n+1}=x\left(t_{n+1}\right)-\bar{x}_{n+1}
$$

and the global error is

$$
\epsilon_{n}=x\left(t_{n}\right)-x_{n}
$$

Obviously, $\epsilon_{n}$ is $\mathscr{F}_{t_{n}}$-measurable because both $x\left(t_{n}\right)$ and $\bar{x}_{n}$ are $\mathscr{F}_{t_{n}}$-measurable.

Theorem 2. Under the assumptions (i)-(iv), there exists a constant $0<\Delta^{*}<1$ such that, for any $0 \leq \Delta \leq \Delta^{*}$, the local error of the compensated split-step $\theta$ method satisfies

$$
\begin{aligned}
& \max _{0 \leq n \leq N-1}\left\|\mathbb{E}\left(\delta_{n+1} \mid \mathscr{F}_{t_{n}}\right)\right\|_{L_{2}} \leq H_{1} \Delta^{3 / 2-1 / p} \quad \text { as } \Delta \longrightarrow 0, \\
& \max _{0 \leq n \leq N-1}\left\|\delta_{n+1}\right\|_{L_{2}} \leq H_{2} \Delta^{1-1 / p} \text { as } \Delta \longrightarrow 0
\end{aligned}
$$

where the constants $H_{1}$ and $H_{2}$ are independent of $\Delta$.

Proof. From (14a), (14b), (15a), and (15b), we have

$$
\begin{gathered}
\delta_{n+1}=\int_{t_{n}}^{t_{n+1}}\left[f_{\lambda}\left(x(s), x(s-\tau(s)), \gamma_{N(s)+1}\right)\right. \\
\left.-f_{\lambda}\left(x\left(t_{n}\right), x\left(t_{m}\right), \gamma_{N\left(t_{n}\right)+1}\right)\right] \mathrm{d} s \\
+\theta \int_{t_{n}}^{t_{n+1}}\left[f_{\lambda}\left(x\left(t_{n}\right), x\left(t_{m}\right), \gamma_{N\left(t_{n}\right)+1}\right)\right. \\
\left.\quad-f_{\lambda}\left(\bar{x}_{n}^{*}, x\left(t_{\bar{m}}\right), \gamma_{N\left(t_{n}\right)+1}\right)\right] \mathrm{d} s \\
+\int_{t_{n}}^{t_{n+1}}[g(x(s), x(s-\tau(s))) \\
\left.+g\left(\bar{x}_{n}^{*}, x\left(t_{m}\right)\right)\right] \mathrm{d} W(s) \\
+\int_{t_{n}}^{t_{n+1}}\left[h\left(x(s), x(s-\tau(s)), \gamma_{N(s)+1}\right)\right. \\
\left.-h\left(\bar{x}_{n}^{*}, x\left(t_{m}\right), \gamma_{N\left(t_{n}\right)+1}\right)\right] \mathrm{d} \bar{N}(s) .
\end{gathered}
$$

Thus

$$
\begin{aligned}
\left|\mathbb{E}\left[\left(x\left(t_{n+1}\right)-\bar{x}_{n+1}\right) \mid \mathscr{F}_{t_{n}}\right]\right| \\
\leq \mid \mathbb{E}\left(\int _ { t _ { n } } ^ { t _ { n + 1 } } \left(f_{\lambda}\left(x(s), x(s-\tau(s)), \gamma_{N(s)+1}\right)\right.\right. \\
\left.\left.\quad-f_{\lambda}\left(x\left(t_{n}\right), x\left(t_{m}\right), \gamma_{N\left(t_{n}\right)+1}\right)\right) \mathrm{d} s \mid \mathscr{F}_{t_{n}}\right) \mid \\
+\theta \mid \mathbb{E}\left(\int _ { t _ { n } } ^ { t _ { n + 1 } } \left(f_{\lambda}\left(x\left(t_{n}\right), x\left(t_{m}\right), \gamma_{N\left(t_{n}\right)+1}\right)\right.\right. \\
\left.\left.\quad-f_{\lambda}\left(\bar{x}_{n}^{*}, x\left(t_{\bar{m}}\right), \gamma_{N\left(t_{n}\right)+1}\right)\right) \mathrm{d} s \mid \mathscr{F}_{t_{n}}\right) \mid
\end{aligned}
$$




$$
\begin{aligned}
& \leq(1+\lambda) K_{2} \int_{t_{n}}^{t_{n+1}} \mathbb{E}\left(\left|x(s)-x\left(t_{n}\right)\right| \mid \mathscr{F}_{t_{n}}\right) \mathrm{d} s \\
& +(1+\lambda) K_{2} \int_{t_{n}}^{t_{n+1}} \mathbb{E}\left(\left|x(s-\tau(s))-x\left(t_{m}\right)\right|\right) \mathrm{d} s \\
& +\theta(1+\lambda) K_{2} \int_{t_{n}}^{t_{n+1}} \mathbb{E}\left(\left|\bar{x}_{n}^{*}-x\left(t_{n}\right)\right| \mid \mathscr{F}_{t_{n}}\right) \mathrm{d} s \\
& +\theta(1+\lambda) K_{2} \int_{t_{n}}^{t_{n+1}} \mathbb{E}\left(\left|x\left(t_{\bar{m}}\right)-x\left(t_{m}\right)\right|\right) \mathrm{d} s \\
& +\lambda K_{2}\left|\mathbb{E}\left(\int_{t_{n}}^{t_{n+1}}\left(\gamma_{N(s)+1}-\gamma_{N\left(t_{n}\right)+1}\right) \mathrm{d} s \mid \mathscr{F}_{t_{n}}\right)\right| .
\end{aligned}
$$

To estimate $\mathbb{E}\left(\left|x(s-\tau(s))-x\left(t_{m}\right)\right|\right)$, let us consider the following five possible cases.

(1) If $s-\tau(s) \geq[n \Delta-\tau(n \Delta)] \Delta \geq 0$, then

$$
\begin{aligned}
s- & \tau(s)-[n \Delta-\tau(n \Delta)] \Delta \\
& \leq s-\tau(s)+\tau(n \Delta)-(n-1) \Delta \leq\left(K_{1}+2\right) \Delta .
\end{aligned}
$$

From Theorem 1, it is obtained that

$$
\begin{aligned}
& \mathbb{E}\left(\left|x(s-\tau(s))-x\left(t_{m}\right)\right|\right) \\
& \quad \leq C_{2}^{1 / 2}|s-\tau(s)-[n \Delta-\tau(n \Delta)] \Delta|^{1 / 2} \\
& \quad \leq C_{2}^{1 / 2}\left(2+K_{1}\right)^{1 / 2} \Delta^{1 / 2} .
\end{aligned}
$$

(2) If $[n \Delta-\tau(n \Delta)] \Delta \geq s-\tau(s) \geq 0$, then

$$
\begin{aligned}
& {[n \Delta-\tau(n \Delta)] \Delta-s+\tau(s)} \\
& \quad \leq n \Delta-\tau(n \Delta)-s+\tau(s) \leq K_{1} \Delta .
\end{aligned}
$$

Thus $\mathbb{E}\left(\left|x(s-\tau(s))-x\left(t_{m}\right)\right|\right) \leq C_{2}^{1 / 2} K_{1}^{1 / 2} \Delta^{1 / 2}$.

(3) If $s-\tau(s) \leq 0$ and $[n \Delta-\tau(n \Delta)] \Delta \leq 0$, then

$$
|s-\tau(s)-[n \Delta-\tau(n \Delta)] \Delta| \leq\left(K_{1}+2\right) \Delta .
$$

Therefore,

$$
\begin{aligned}
\mathbb{E} & \left(\left|x(s-\tau(s))-x\left(t_{m}\right)\right|\right) \\
& =\mathbb{E}\left(\left|\xi(s-\tau(s))-\xi\left(t_{m}\right)\right|\right) \\
& \leq K_{4}^{1 / 2}|s-\tau(s)-[n \Delta+\tau(n \Delta)] \Delta|^{1 / 2} \\
& \leq K_{4}^{1 / 2}\left(2+K_{1}\right)^{1 / 2} \Delta^{1 / 2} .
\end{aligned}
$$

(4) If $s-\tau(s) \geq 0$ and $[n \Delta-\tau(n \Delta)] \Delta \leq 0$, then

$$
\begin{aligned}
s-\tau(s) & \leq s-\tau(s)-[n \Delta+\tau(n \Delta)] \Delta \\
& \leq\left(K_{1}+2\right) \Delta, \\
-[n \Delta+\tau(n \Delta)] \Delta & \leq s-\tau(s)-[n \Delta+\tau(n \Delta)] \Delta \\
& \leq\left(K_{1}+2\right) \Delta .
\end{aligned}
$$

Thus

$$
\begin{aligned}
\mathbb{E} & \left(\left|x(s-\tau(s))-x\left(t_{m}\right)\right|\right) \\
& =\mathbb{E}\left(\left|x(s-\tau(s))-\xi\left(t_{m}\right)\right|\right) \\
& \leq \mathbb{E}\left(|x(s-\tau(s))-\xi(0)|+\left|\xi(0)-\xi\left(t_{m}\right)\right|\right) \\
& \leq K_{4}^{1 / 2}\left(|s-\tau(s)|^{1 / 2}+|[n \Delta+\tau(n \Delta)] \Delta|^{1 / 2}\right) \\
& \leq 2 K_{4}^{1 / 2}\left(2+K_{1}\right)^{1 / 2} \Delta^{1 / 2} .
\end{aligned}
$$

(5) If $s-\tau(s) \leq 0$ and $[n \Delta-\tau(n \Delta)] \Delta \geq 0$, then

$$
\begin{aligned}
-[s-\tau(s)] & \leq[n \Delta+\tau(n \Delta)]-[s-\tau(s)] \Delta \\
& \leq\left(K_{1}+2\right) \Delta, \\
{[n \Delta+\tau(n \Delta)] \Delta } & \leq[n \Delta+\tau(n \Delta)]-[s-\tau(s)] \\
& \leq\left(K_{1}+2\right) \Delta .
\end{aligned}
$$

Thus

$$
\begin{aligned}
\mathbb{E} & \left(\left|x(s-\tau(s))-x\left(t_{m}\right)\right|\right) \\
& =\mathbb{E}\left(\left|\xi(s-\tau(s))-x\left(t_{m}\right)\right|\right) \\
& \leq \mathbb{E}\left(|\xi(s-\tau(s))-\xi(0)|+\left|\xi(0)-x\left(t_{m}\right)\right|\right) \\
& \leq 2 K_{4}^{1 / 2}\left(2+K_{1}\right)^{1 / 2} \Delta^{1 / 2} .
\end{aligned}
$$

Combining the above five cases, it can be concluded that there exists a constant $K$ such that $\mathbb{E}\left(\left|x(s-\tau(s))-x\left(t_{m}\right)\right|\right) \leq K \Delta^{1 / 2}$.

Similarly, it can be proved that there exists a constant $K^{i}$ such that $\mathbb{E}\left(\left|x(s-\tau(s))-x\left(t_{\bar{m}}\right)\right|\right) \leq K^{\prime} \Delta^{1 / 2}$. Therefore,

$$
\begin{aligned}
\mathbb{E}\left(\left|x\left(t_{\bar{m}}\right)-x\left(t_{m}\right)\right|\right) \leq & \mathbb{E}\left(\left|x(s-\tau(s))-x\left(t_{\bar{m}}\right)\right|\right) \\
& +\mathbb{E}\left(\left|x(s-\tau(s))-x\left(t_{m}\right)\right|\right) \\
\leq & \left(K+K^{\prime}\right) \Delta^{1 / 2} .
\end{aligned}
$$

From (15a) and Theorem 1, we have, for any $\Delta<1 /$ $\left[K_{3} \theta(1+\lambda)\right]$, that there exists a constant $K_{6}$ such that

$$
\begin{aligned}
\mathbb{E}\left(\left|\bar{x}_{n}^{*}\right| \mid \mathscr{F}_{t_{n}}\right) & \\
=\mathbb{E}\left(\mid x\left(t_{n}\right)+\right. & {\left[\theta f_{\lambda}\left(\bar{x}_{n}^{*}, x\left(t_{\bar{m}}\right), \gamma_{N\left(t_{n}\right)+1}\right)\right.} \\
& \left.+(1-\theta) f_{\lambda}\left(x\left(t_{n}\right), x\left(t_{m}\right), \gamma_{N\left(t_{n}\right)+1}\right)\right] \Delta|| \\
\left.\mathscr{F}_{t_{n}}\right) \leq & K_{6} .
\end{aligned}
$$


Hence,

$$
\begin{aligned}
\mathbb{E}\left(\left|\bar{x}_{n}^{*}-x\left(t_{n}\right)\right| \mid \mathscr{F}_{t_{n}}\right) & \\
= & \mathbb{E}\left(\mid\left[\theta f_{\lambda}\left(\bar{x}_{n}^{*}, x\left(t_{\bar{m}}\right), \gamma_{N\left(t_{n}\right)+1}\right)\right.\right. \\
& \left.\left.\quad+(1-\theta) f_{\lambda}\left(x\left(t_{n}\right), x\left(t_{m}\right), \gamma_{N\left(t_{n}\right)+1}\right)\right] \Delta|| \mathscr{F}_{t_{n}}\right) \\
\leq & {\left[\theta(1+\lambda) K_{3}\left(1+\mathbb{E}\left(\left|\bar{x}_{n}^{*}\right| \mid \mathscr{F}_{t_{n}}\right)+\mathbb{E}\left(\left|x\left(t_{\bar{m}}\right)\right| \mid \mathscr{F}_{t_{n}}\right)\right)\right.} \\
& +(1-\theta)(1+\lambda) K_{3} \\
& \quad\left(1+\mathbb{E}\left(\left|x\left(t_{n}\right)\right| \mid \mathscr{F}_{t_{n}}\right)+\mathbb{E}\left(\left|x\left(t_{m}\right)\right| \mid \mathscr{F}_{t_{n}}\right)\right) \\
& \left.+\lambda K_{3} \mathbb{E}\left(\left|\gamma_{N\left(t_{n}\right)+1}\right| \mid \mathscr{F}_{t_{n}}\right)\right] \Delta \\
\leq & (1+\lambda) K_{3}\left(1+2 C_{1}+K_{6} \theta-C_{1} \theta\right) \\
& \left.+\lambda K_{3} \mathbb{E}\left(\left|\gamma_{N\left(t_{n}\right)+1}\right| \mid \mathscr{F}_{t_{n}}\right)\right] \Delta .
\end{aligned}
$$

Moreover, it is known from Theorem 3.4 in [12] that

$$
\mathbb{E}\left(\int_{t_{n}}^{t_{n+1}}\left|\gamma_{N(s)+1}-\gamma_{N\left(t_{n}\right)+1}\right|^{2} \mathrm{~d} s\right) \leq C \Delta^{2-2 / p} .
$$

Thus,

$$
\begin{aligned}
& \left|\mathbb{E}\left(\int_{t_{n}}^{t_{n+1}}\left(\gamma_{N(s)+1}-\gamma_{N\left(t_{n}\right)+1}\right) \mathrm{d} s \mid \mathscr{F}_{t_{n}}\right)\right| \\
& \quad \leq C^{1 / 2} \Delta^{3 / 2-1 / p} .
\end{aligned}
$$

Summing up the above conclusions, we can obtain that, for any $\Delta<1 /\left[K_{3} \theta(1+\lambda)\right]$, there exists a constant $H_{1}$ such that

$$
\mathbb{E}\left|\mathbb{E}\left[\left(x\left(t_{n+1}\right)-\bar{x}_{n+1}\right) \mid \mathscr{F}_{t_{n}}\right]\right|^{2} \leq H_{1}^{2} \Delta^{3-2 / p} .
$$

Otherwise, for any $\Delta<1 /\left[4 K_{3}^{2} \theta^{2}\left(1+\lambda^{2}\right)\right]$, there exists a constant $\mathrm{H}_{2}$ such that

$$
\begin{array}{r}
\mathbb{E}\left(\left|x\left(t_{n+1}\right)-\bar{x}_{n+1}\right|^{2}\right) \\
\leq 4 \mathbb{E}\left(\mid \int_{t_{n}}^{t_{n+1}}\left[f_{\lambda}\left(x(s), x(s-\tau(s)), \gamma_{N(s)+1}\right)\right.\right. \\
\left.\left.\quad-f_{\lambda}\left(x\left(t_{n}\right), x\left(t_{m}\right), \gamma_{N\left(t_{n}\right)+1}\right)\right]\left.\mathrm{d} s\right|^{2}\right) \\
+4 \theta \mathbb{E}\left(\mid \int_{t_{n}}^{t_{n+1}}\left[f_{\lambda}\left(x\left(t_{n}\right), x\left(t_{m}\right), \gamma_{N\left(t_{n}\right)+1}\right)\right.\right. \\
\left.\left.\quad-f_{\lambda}\left(\bar{x}_{n}^{*}, x\left(t_{\bar{m}}\right), \gamma_{N\left(t_{n}\right)+1}\right)\right]\left.\mathrm{d} s\right|^{2}\right)
\end{array}
$$

$$
\begin{gathered}
+4 \mathbb{E}\left(\mid \int_{t_{n}}^{t_{n+1}}[g(x(s), x(s-\tau(s)))\right. \\
\left.\left.-g\left(\bar{x}_{n}^{*}, x\left(t_{m}\right)\right)\right]\left.\mathrm{d} W(s)\right|^{2}\right) \\
+4 \mathbb{E}\left(\mid \int_{t_{n}}^{t_{n+1}}\left[h\left(x(s), x(s-\tau(s)), \gamma_{N(s)+1}\right)\right.\right.
\end{gathered}
$$$$
\left.\left.-h\left(\bar{x}_{n}^{*}, x\left(t_{m}\right), \gamma_{N\left(t_{n}\right)+1}\right)\right]\left.\mathrm{d} N(s)\right|^{2}\right)
$$$$
\leq 4 K_{2}^{2} \Delta \int_{t_{n}}^{t_{n+1}}\left[(1+\lambda) \mathbb{E}\left(\left|x(s)-x\left(t_{n}\right)\right|^{2}\right)\right.
$$$$
\left.+(1+\lambda) \mathbb{E}\left(\left|x(s-\tau(s))-x\left(t_{m}\right)\right|^{2}\right)\right] \mathrm{d} s
$$$$
+4 \theta K_{2}^{2} \Delta \int_{t_{n}}^{t_{n+1}}\left[(1+\lambda) \mathbb{E}\left(\left|x\left(t_{n}\right)-\bar{x}_{n}^{*}\right|^{2}\right)\right.
$$$$
\left.+(1+\lambda) \mathbb{E}\left(\left|x\left(t_{m}\right)-x\left(t_{\bar{m}}\right)\right|^{2}\right)\right] \mathrm{d} s
$$

$$
+4 K_{2}^{2}(1+\lambda) \int_{t_{n}}^{t_{n+1}}\left[\mathbb{E}\left(\left|x(s)-\bar{x}_{n}^{*}\right|^{2}\right)\right.
$$

$$
\left.+\mathbb{E}\left(\left|x(s-\tau(s))-x\left(t_{m}\right)\right|^{2}\right)\right] \mathrm{d} s
$$

$$
+4 K_{2}^{2} \lambda(1+\Delta) \int_{t_{n}}^{t_{n+1}}\left[\mathbb{E}\left(\left|\gamma_{N(s)+1}-\gamma_{N\left(t_{n}\right)+1}\right|^{2}\right)\right] \mathrm{d} s
$$$$
\leq H_{2}^{2} \Delta^{2(1-1 / p)} \text {. }
$$

Theorem 3. Under assumptions (i)-(iv), there exists a constant $0<\Delta^{*}<1$ such that, for any $0 \leq \Delta \leq \Delta^{*}$, the global error of the compensated split-step $\theta$ method satisfies

$$
\max _{0 \leq n \leq N-1}\left\|\epsilon_{n+1}^{2}\right\|_{L_{2}} \leq H_{3} \Delta^{1 / 2-1 / p} \text { as } \Delta \longrightarrow 0,
$$

where $\mathrm{H}_{3}$ is independent of $\Delta$.

Proof. From the definitions of $\delta_{n}$ and $\epsilon_{n}$, we have

$$
\epsilon_{n+1}=\epsilon_{n}+u_{n}+\delta_{n+1} \text {, }
$$

where

$$
\begin{aligned}
u_{n}= & \theta\left[f_{\lambda}\left(\bar{x}_{n}^{*}, x\left(t_{\bar{m}}\right), \gamma_{N\left(t_{n}\right)+1}\right)-f_{\lambda}\left(x_{n}^{*}, x_{\bar{m}}, \gamma_{N\left(t_{n}\right)+1}\right)\right] \Delta \\
+ & (1-\theta)\left[f_{\lambda}\left(x\left(t_{n}\right), x\left(t_{m}\right), \gamma_{N\left(t_{n}\right)+1}\right)\right. \\
& \left.-f_{\lambda}\left(x_{n}, x_{m}, \gamma_{N\left(t_{n}\right)+1}\right)\right] \Delta \\
+ & {\left[g\left(\bar{x}_{n}^{*}, x\left(t_{m}\right)\right)-g\left(x_{n}^{*}, x_{m}\right)\right] \Delta W_{n} } \\
+ & {\left[h\left(\bar{x}_{n}^{*}, x\left(t_{m}\right), \gamma_{N\left(t_{n}\right)+1}\right)-h\left(x_{n}^{*}, x_{m}, \gamma_{N\left(t_{n}\right)+1}\right)\right] \Delta \bar{N}_{n} . }
\end{aligned}
$$


From (14a), (15a), and assumption (ii) it can be obtained that, for any $\Delta<1 /\left[K_{2} \theta(1+\lambda)\right]$,

$$
\begin{aligned}
& \mathbb{E}\left(\left|\bar{x}_{n}^{*}-x_{n}^{*}\right| \mid \mathscr{F}_{t_{n}}\right) \\
& =\mathbb{E}\left(\mid x\left(t_{n}\right)-x_{n}\right. \\
& +\theta\left[f_{\lambda}\left(\bar{x}_{n}^{*}, x\left(t_{\bar{m}}\right), \gamma_{N\left(t_{n}\right)+1}\right)\right. \\
& \left.\quad-f_{\lambda}\left(x_{n}^{*}, x_{\bar{m}}, \gamma_{N\left(t_{n}\right)+1}\right)\right] \Delta \\
& +(1-\theta)\left[f_{\lambda}\left(x\left(t_{n}\right), x\left(t_{m}\right), \gamma_{N\left(t_{n}\right)+1}\right)\right. \\
& \left.\left.\quad-f_{\lambda}\left(x_{n}, x_{m}, \gamma_{N\left(t_{n}\right)+1}\right)\right] \Delta|| \mathscr{F}_{t_{n}}\right) \\
& \leq \frac{1}{1-} K_{2} \theta(1+\lambda) \Delta \\
& \quad\left[\left(1+K_{2}(1-\theta)(1+\lambda) \Delta\right) \mathbb{E}\left(\left|\epsilon_{n}\right| \mid \mathscr{F}_{t_{n}}\right)\right. \\
& +K_{2} \theta(1+\lambda) \Delta \mathbb{E}\left(\left|\epsilon_{\bar{m}}\right|\right) \\
& \left.\quad+K_{2}(1-\theta)(1+\lambda) \Delta \mathbb{E}\left(\left|\epsilon_{m}\right|\right)\right]
\end{aligned}
$$

and, for any $\Delta<1 /\left[6 K_{2}^{2} \theta^{2}\left(1+\lambda^{2}\right)\right]$,

$$
\begin{aligned}
\mathbb{E}\left(\left|\bar{x}_{n}^{*}-x_{n}^{*}\right|^{2}\right) & \\
=\mathbb{E}\left(\mid x\left(t_{n}\right)-x_{n}\right. & +\theta\left[f_{\lambda}\left(\bar{x}_{n}^{*}, x\left(t_{\bar{m}}\right), \gamma_{N\left(t_{n}\right)+1}\right)\right. \\
& \left.-f_{\lambda}\left(x_{n}^{*}, x_{\bar{m}}, \gamma_{N\left(t_{n}\right)+1}\right)\right] \Delta \\
& +(1-\theta)\left[f_{\lambda}\left(x\left(t_{n}\right), x\left(t_{m}\right), \gamma_{N\left(t_{n}\right)+1}\right)\right. \\
& \left.\left.-f_{\lambda}\left(x_{n}, x_{m}, \gamma_{N\left(t_{n}\right)+1}\right)\right]\left.\Delta\right|^{2}\right) \\
1- & 6 K_{2}^{2} \theta^{2}\left(1+\lambda^{2}\right) \Delta \\
& {\left[3\left(1+2 K_{2}^{2}(1-\theta)^{2}\left(1+\lambda^{2}\right) \Delta^{2}\right) \mathbb{E}\left(\left|\epsilon_{n}\right|^{2}\right)\right.} \\
& +6 K_{2}^{2} \theta^{2}\left(1+\lambda^{2}\right) \Delta^{2} \mathbb{E}\left(\left|\epsilon_{\bar{m}}\right|^{2}\right) \\
& \left.+6 K_{2}^{2}(1-\theta)^{2}\left(1+\lambda^{2}\right) \Delta^{2} \mathbb{E}\left(\left|\epsilon_{m}\right|^{2}\right)\right] .
\end{aligned}
$$

Since $\mathbb{E}\left(\Delta W_{n}\right)=0, \mathbb{E}\left(\Delta W_{n}\right)^{2}=\Delta, \mathbb{E}\left(\Delta \bar{N}_{n}\right)=0$, and $\mathbb{E}\left(\Delta \bar{N}_{n}\right)^{2}=\lambda \Delta$, we known from assumption (ii) that

$$
\begin{aligned}
\left|\mathbb{E}\left(u_{n} \mid \mathscr{F}_{t_{n}}\right)\right| \\
\leq K_{2} \theta(1+\lambda) \Delta\left[\mathbb{E}\left(\left|\bar{x}_{n}^{*}-x_{n}^{*}\right| \mid \mathscr{F}_{t_{n}}\right)+\mathbb{E}\left|\epsilon_{\bar{m}}\right|\right] \\
\quad+K_{2}(1-\theta)(1+\lambda) \Delta\left[\mathbb{E}\left(\left|\epsilon_{n}\right| \mid \mathscr{F}_{t_{n}}\right)+\mathbb{E}\left|\epsilon_{m}\right|\right] \\
\leq K_{7} \Delta\left[\mathbb{E}\left(\left|\epsilon_{n}\right| \mid \mathscr{F}_{t_{n}}\right)+\mathbb{E}\left(\left|\epsilon_{m}\right|\right)+\mathbb{E}\left(\left|\epsilon_{\bar{m}}\right|\right)\right],
\end{aligned}
$$

$$
\begin{aligned}
\mathbb{E}\left(\left|u_{n}\right|^{2}\right) & \\
\leq & 4 K_{2}^{2} \Delta\left(1+\lambda+2 \theta^{2}\left(1+\lambda^{2}\right) \Delta\right) \mathbb{E}\left(\left|\bar{x}_{n}^{*}-x_{n}^{*}\right|^{2}\right) \\
& +8 K_{2}^{2}(1-\theta)^{2}\left(1+\lambda^{2}\right) \Delta^{2} \mathbb{E}\left(\left|\epsilon_{n}\right|^{2}\right) \\
& +8 K_{2}^{2} \theta^{2}\left(1+\lambda^{2}\right) \Delta^{2} \mathbb{E}\left(\left|\epsilon_{\bar{m}}\right|^{2}\right) \\
& +4 K_{2}^{2} \Delta\left(1+\lambda+2(1-\theta)^{2}\left(1+\lambda^{2}\right) \Delta\right) \mathbb{E}\left(\left|\epsilon_{m}\right|^{2}\right) \\
\leq & K_{8} \Delta\left[\mathbb{E}\left(\left|\epsilon_{n}\right|^{2}\right)+\mathbb{E}\left(\left|\epsilon_{\bar{m}}\right|^{2}\right)+\mathbb{E}\left(\left|\epsilon_{m}\right|^{2}\right)\right],
\end{aligned}
$$

where $K_{7}=4 K_{2}(1+\lambda)+3 K_{2}^{2}(1+\lambda)^{2}, K_{8}=4 K_{2}^{2}(1+\lambda+2(1+$ $\left.\left.\lambda^{2}\right)\right)\left(15 K_{2}^{2}\left(1+\lambda^{2}\right)+3\right)+4 K_{2}^{2}\left(1+\lambda+6\left(1+\lambda^{2}\right)\right)$. Hence

$$
\begin{aligned}
\mid \mathbb{E} & \left\langle\epsilon_{n}, u_{n}\right\rangle \mid \\
= & \left|\mathbb{E}\left[\mathbb{E}\left(\left\langle\epsilon_{n}, u_{n}\right\rangle \mid \mathscr{F}_{t_{n}}\right)\right]\right| \\
\leq & \mathbb{E}\left[\left|\epsilon_{n}\right| \cdot\left|\mathbb{E}\left(u_{n} \mid \mathscr{F}_{t_{n}}\right)\right|\right] \\
\leq & {\left[\Delta^{-1}\left(\mathbb{E}\left|\mathbb{E}\left(u_{n} \mid \mathscr{F}_{t_{n}}\right)\right|^{2}\right)\right]^{1 / 2}\left(\Delta \mathbb{E}\left|\epsilon_{n}\right|^{2}\right)^{1 / 2} } \\
\leq & \Delta^{-1}\left(\mathbb{E}\left|\mathbb{E}\left(u_{n} \mid \mathscr{F}_{t_{n}}\right)\right|^{2}\right)+\Delta \mathbb{E}\left(\left|\epsilon_{n}\right|^{2}\right) \\
\leq & K_{7} \Delta\left[\mathbb{E}\left(\left|\epsilon_{n}\right|^{2}\right)+\mathbb{E}\left(\left|\epsilon_{\bar{m}}\right|^{2}\right)+\mathbb{E}\left(\left|\epsilon_{m}\right|^{2}\right)\right] \\
& +\Delta \mathbb{E}\left(\left|\epsilon_{n}\right|^{2}\right) .
\end{aligned}
$$

From Theorem 2, we have

$$
\begin{aligned}
\mid \mathbb{E} & \left\langle\delta_{n+1}, \epsilon_{n}\right\rangle \mid \\
& =\left|\mathbb{E}\left[\mathbb{E}\left(\left\langle\delta_{n+1}, \epsilon_{n}\right\rangle \mid \mathscr{F}_{t_{n}}\right)\right]\right| \\
& \leq \mathbb{E}\left|\left\langle\mathbb{E}\left(\delta_{n+1} \mid \mathscr{F}_{t_{n}}\right), \epsilon_{n}\right\rangle\right| \\
& \leq\left[\Delta^{-1}\left(\mathbb{E}\left|\mathbb{E}\left(\delta_{n+1} \mid \mathscr{F}_{t_{n}}\right)\right|^{2}\right)\right]^{1 / 2}\left(\Delta \mathbb{E}\left|\epsilon_{n}\right|^{2}\right)^{1 / 2} \\
& \leq \Delta^{-1}\left(\mathbb{E}\left|\mathbb{E}\left(\delta_{n+1} \mid \mathscr{F}_{t_{n}}\right)\right|^{2}\right)+\Delta \mathbb{E}\left(\left|\epsilon_{n}\right|^{2}\right) \\
& \leq H_{1} \Delta^{2-2 / p}+\Delta \mathbb{E}\left(\left|\epsilon_{n}\right|^{2}\right) .
\end{aligned}
$$

Combining the above conclusions, we have

$$
\begin{aligned}
& \mathbb{E}\left(\left|\epsilon_{n+1}\right|^{2}\right) \\
& \leq \mathbb{E}\left(\left|\epsilon_{n}\right|^{2}\right)+2 \mathbb{E}\left(\left|\delta_{n+1}\right|^{2}\right)+2 \mathbb{E}\left(\left|u_{n}\right|^{2}\right) \\
& \quad+2\left|\mathbb{E}\left\langle\delta_{n+1}, \epsilon_{n}\right\rangle\right|+2\left|\mathbb{E}\left\langle\epsilon_{n}, u_{n}\right\rangle\right| \\
& \leq\left[1+2\left(1+K_{7}+K_{8}\right) \Delta\right] \mathbb{E}\left(\left|\epsilon_{n}\right|^{2}\right) \\
& \quad+2 \Delta\left(K_{7}+K_{8}\right)\left(\mathbb{E}\left(\left|\epsilon_{[(n \Delta-\tau(n \Delta)) / \Delta]}\right|^{2}\right)\right. \\
& \left.\quad+\mathbb{E}\left(\left|\epsilon_{[((n+1) \Delta-\tau((n+1) \Delta)) / \Delta]}\right|^{2}\right)\right) \\
& +\left(H_{1}+2 H_{2}^{2}\right) \Delta^{2(1-1 / p) .}
\end{aligned}
$$


Additionally, when $-r \leq n \leq 0, \epsilon_{n}=0$. From (45), it can be obtained that the following items are true.

(1) If $[(n \Delta-\tau(n \Delta)) / \Delta] \Delta \leq 0$ and $[((n+1) \Delta-\tau((n+$ 1) $\Delta)) / \Delta] \Delta \leq 0$, we have

$$
\begin{aligned}
\mathbb{E}\left(\left|\epsilon_{n+1}\right|^{2}\right) & \\
\leq & {\left[1+2\left(1+K_{7}+K_{8}\right) \Delta\right] \mathbb{E}\left|\epsilon_{n}\right|^{2} } \\
& +\left(H_{1}+2 H_{2}^{2}\right) \Delta^{2(1-1 / p)} \\
\leq & \left(H_{1}+2 H_{2}^{2}\right) \Delta^{1-2 / p} \sum_{i=0}^{n}\left[1+2\left(1+K_{7}+K_{8}\right) \Delta\right]^{i} \\
\leq & \left(H_{1}+2 H_{2}^{2}\right) \frac{e^{2\left(1+K_{7}+K_{8}\right) T}-1}{2\left(1+K_{7}+K_{8}\right)} \Delta^{1-2 / p} .
\end{aligned}
$$

(2) If $[(n \Delta-\tau(n \Delta)) / \Delta] \Delta \geq 0$ and $[((n+1) \Delta-\tau((n+$ 1) $\Delta)) / \Delta] \Delta \geq 0$, we have

$$
\begin{aligned}
\mathbb{E}\left(\left|\epsilon_{n+1}\right|^{2}\right) \leq & {\left[1+2\left(1+3 K_{7}+3 K_{8}\right) \Delta\right] \max _{0 \leq i \leq n}\left(\mathbb{E}\left|\epsilon_{i}\right|^{2}\right) } \\
& +\left(H_{1}+2 H_{2}^{2}\right) \Delta^{2(1-1 / p)} \\
\leq & \left(H_{1}+2 H_{2}^{2}\right) \frac{e^{2\left(1+3 K_{7}+3 K_{8}\right) T}-1}{2\left(1+3 K_{7}+3 K_{8}\right)} \Delta^{1-2 / p} .
\end{aligned}
$$

(3) If $([(n \Delta-\tau(n \Delta)) / \Delta] \Delta)([((n+1) \Delta-\tau((n+1) \Delta)) / \Delta] \Delta) \leq$ 0 , we have

$$
\begin{aligned}
\mathbb{E}\left(\left|\epsilon_{n+1}\right|^{2}\right) \leq & {\left[1+2\left(1+2 K_{7}+2 K_{8}\right) \Delta\right] \max _{0 \leq i \leq n}\left(\mathbb{E}\left|\epsilon_{i}\right|^{2}\right) } \\
& +\left(H_{1}+2 H_{2}^{2}\right) \Delta^{2(1-1 / p)} \\
\leq & \left(H_{1}+2 H_{2}^{2}\right) \frac{e^{2\left(1+2 K_{7}+2 K_{8}\right) T}-1}{2\left(1+2 K_{7}+2 K_{8}\right)} \Delta^{1-2 / p} .
\end{aligned}
$$

Combining the above three cases, it can be obtained that

$$
\max _{0 \leq n \leq N-1}\left(\mathbb{E}\left|\epsilon_{n+1}\right|^{2}\right)^{1 / 2} \leq H_{3} \Delta^{1 / 2-1 / p}
$$

where $H_{3}=\left(\left[\left(C_{3}+2 C_{4}^{2}\right)\left(e^{2\left(1+3 K_{7}+3 K_{8}\right) T}-1\right)\right] /\left[2\left(1+K_{7}+\right.\right.\right.$ $\left.\left.\left.K_{8}\right)\right]\right)^{1 / 2}$.

\section{Stability of the Analytical and Numerical Solutions for the SDEVDRJM}

In this section, we will discuss the stability of the analytical solutions of the SDEVDRJM and the numerical method introduced in Section 2.

Consider the following scalar test equation:

$$
\begin{aligned}
\mathrm{d} x(t)= & \left(a_{1} x(t)+a_{2} x(t-\tau(t))\right) \mathrm{d} t \\
& +\left(b_{1} x(t)+b_{2} x(t-\tau(t))\right) \mathrm{d} W(t) \\
& +\gamma(t) x(t) \mathrm{d} N(t),
\end{aligned}
$$

where $a_{i}$ and $b_{i}$ are real constants. Define $\gamma(t)=\gamma_{N(t)+1}=$ $\sum_{j} \gamma_{j+1} 1_{\left[\tau_{j}, \tau_{j+1}\right]}(t)$, where $\tau_{0}=0$ and $\tau_{j}(j=1,2, \ldots)$ are the jump times.
4.1. Mean-Square Stability of the Analytical Solutions. In what follows, we give some sufficient conditions on the stability property of the analytical solutions of (50).

Theorem 4. Assume that the constants $a_{1}, a_{2}, b_{1}, b_{2}$, and $\lambda$ and the random variable $\gamma$ satify

$$
\begin{aligned}
2 a_{1}+ & b_{1}^{2}+2 \lambda \mathbb{E}(\gamma)+\lambda \mathbb{E}\left(|\gamma|^{2}\right)+2\left|a_{2}\right|+b_{2}^{2} \\
& +2\left|b_{1}\right|\left|b_{2}\right|<0 .
\end{aligned}
$$

Then solution of (50) is mean-square stable. That is,

$$
\lim _{t \rightarrow \infty} \mathbb{E}\left(|x(t)|^{2}\right)=0 \text {. }
$$

Proof. For any $t>0$ and $\delta>0$, it follows from Itô formula that

$$
\begin{aligned}
|x(t+\delta)|^{2} & \\
= & |x(t)|^{2}+\int_{t}^{t+\delta}\left(2\left\langle x(s), a_{1} x(s)+a_{2} x(s-\tau(s))\right\rangle\right. \\
& \left.+\left|b_{1} x(s)+b_{2} x(s-\tau(s))\right|^{2}\right) \mathrm{d} s \\
& +\int_{t}^{t+\delta} 2\left\langle x(s), b_{1} x(s)+b_{2} x(s-\tau(s))\right\rangle \mathrm{d} W(s) \\
& +\int_{t}^{t+\delta}\left(2\langle x(s), \gamma(s) x(s)\rangle+|\gamma(s) x(s)|^{2}\right) \mathrm{d} \bar{N}(s) \\
& +\lambda \int_{t}^{t+\delta}\left(2\langle x(s), \gamma(s) x(s)\rangle+|\gamma(s) x(s)|^{2}\right) \mathrm{d} s
\end{aligned}
$$

Taking the expectation yields

$$
\begin{gathered}
\mathbb{E}\left(|x(t+\delta)|^{2}\right)=\mathbb{E}\left(|x(t)|^{2}\right) \\
+\int_{t}^{t+\delta}\left(2 a_{1} \mathbb{E}\left(|x(s)|^{2}\right)\right. \\
+2 a_{2} \mathbb{E}(|x(s)||x(s-\tau(s))|) \\
\left.+\mathbb{E}\left(\left|b_{1} x(s)+b_{2} x(s-\tau(s))\right|^{2}\right)\right) \mathrm{d} s \\
+\lambda \int_{t}^{t+\delta}\left(2 \mathbb{E}\left(\gamma(s)|x(s)|^{2}\right)\right. \\
\left.+\mathbb{E}\left(|\gamma(s) x(s)|^{2}\right)\right) \mathrm{d} s
\end{gathered}
$$




$$
\begin{gathered}
\leq \mathbb{E}\left(|x(t)|^{2}\right) \\
+\int_{t}^{t+\delta}\left[\left(2 a_{1}+b_{1}^{2}+2 \lambda \mathbb{E}(\gamma)+\lambda \mathbb{E}\left(|\gamma|^{2}\right)\right)\right. \\
\cdot \mathbb{E}\left(|x(s)|^{2}\right) \\
+\left(2\left|a_{2}\right|+b_{2}^{2}+2\left|b_{1}\right|\left|b_{2}\right|\right) \\
\left.\cdot \sup _{-r \leq u \leq s} \mathbb{E}\left(|x(u)|^{2}\right)\right] \mathrm{d} s .
\end{gathered}
$$

Let $v(t)=\mathbb{E}\left(|x(t)|^{2}\right), \alpha=2 a_{1}+b_{1}^{2}+2 \lambda \mathbb{E}(\gamma)+\lambda \mathbb{E}\left(|\gamma|^{2}\right)$, and $\beta=2\left|a_{2}\right|+b_{2}^{2}+2\left|b_{1}\right|\left|b_{2}\right|$. It is obtained that

$$
\begin{aligned}
D^{+} v(t) & =\underset{\delta \searrow 0}{\lim \sup } \frac{v(t+\delta)-v(t)}{\delta} \\
& \leq \alpha v(t)+\beta \sup _{-r \leq u \leq t} v(u)
\end{aligned}
$$

From (51), we know $0<\beta<-\alpha$. Furthermore, according to lemma 1.1 in [19], there exist $k$ and $k^{\prime}$ such that

$$
v(t) \leq k e^{-k^{\prime} t}
$$

Thus, $v(t) \rightarrow 0$ when $t \rightarrow \infty$.

4.2. Mean-Square Stability of the Numerical Solutions. Applying the compensated split-step $\theta$ method to the test equation yields

$$
\begin{aligned}
x_{n}^{*}=x_{n}+[ & \theta\left(a_{1} x_{n}^{*}+a_{2} x_{\bar{m}}\right)+(1-\theta)\left(a_{1} x_{n}+a_{2} x_{m}\right) \\
& \left.+\lambda \gamma_{N\left(t_{n}\right)+1} x_{n}\right] \Delta \\
x_{n+1}=x_{n}^{*}+ & \left(b_{1} x_{n}^{*}+b_{2} x_{m}\right) \Delta W_{n}+\gamma_{N\left(t_{n}\right)+1} x_{n}^{*} \Delta \bar{N}_{n} .
\end{aligned}
$$

Note that a numerical method is said to be mean-square stable (MS-stable) if there exists a constant $\Delta^{*}>0$ such that $\lim _{n \rightarrow \infty} \mathbb{E}\left(\left|x_{n}\right|^{2}\right)=0$ for all $\Delta \in\left(0, \Delta^{*}\right)$ and a numerical method is said to be general mean-square stable (GMSstable) if $\lim _{n \rightarrow \infty} \mathbb{E}\left(\left|x_{n}\right|^{2}\right)=0$ holds for every time step size $\Delta>0$. For notational simplicity, let

$$
\begin{aligned}
A= & \left(b_{1}^{2}+\lambda \mathbb{E}\left(\gamma^{2}\right)+\left|b_{1} b_{2}\right|\right) \\
& \cdot\left[(1-\theta)^{2} a_{1}^{2}+\lambda^{2} \mathbb{E}\left(\gamma^{2}\right)+\left|a_{2}\right|^{2}+2(1-\theta) a_{1} \lambda \mathbb{E}(\gamma)\right. \\
& \left.+2\left|a_{2}\right|\left((1-\theta)\left|a_{1}\right|+\lambda \mathbb{E}(|\gamma|)\right)\right]+\theta^{2} a_{1}^{2}\left(b_{2}^{2}+\left|b_{1} b_{2}\right|\right),
\end{aligned}
$$

$$
\begin{aligned}
B= & (1-2 \theta) a_{1}^{2}+\lambda^{2} \mathbb{E}\left(\gamma^{2}\right)+2(1-\theta) a_{1} \lambda \mathbb{E}(\gamma) \\
& +2\left|a_{2}\right|\left((1-\theta)\left|a_{1}\right|+\lambda \mathbb{E}(|\gamma|)\right)+\left|a_{2}\right|^{2} \\
& +2\left(b_{1}^{2}+\lambda \mathbb{E}\left(\gamma^{2}\right)+\left|b_{1} b_{2}\right|\right)\left((1-\theta) a_{1}+\lambda \mathbb{E}(\gamma)+2\left|a_{2}\right|\right) \\
& -2 a_{1} \theta\left(b_{2}^{2}+\left|b_{1} b_{2}\right|\right) \\
\widehat{\Delta}= & B^{2}-4 A\left[2 a_{1}+b_{1}^{2}+2 \lambda \mathbb{E}(\gamma)\right. \\
& \left.+\lambda \mathbb{E}\left(\gamma^{2}\right)+2\left|a_{2}\right|+b_{2}^{2}+2\left|b_{1}\right|\left|b_{2}\right|\right] .
\end{aligned}
$$

Theorem 5. Assume that condition (51) holds. Then we have the following conclusions.

(i) If $A=0$ and $B \leq 0$ or $A<0$ and $B<2\left(A\left[2 a_{1}+b_{1}^{2}+\right.\right.$ $\left.\left.2 \lambda \mathbb{E}(\gamma)+\lambda \mathbb{E} \gamma^{2}+2\left|a_{2}\right|+b_{2}^{2}+2\left|b_{1}\right|\left|b_{2}\right|\right]\right)^{1 / 2}$, then the compensated split-step $\theta$ method for (50) is GMS-stable.

(ii) If $A=0$ and $B>0$, then there exists a constant $\Delta_{0}>0$ such that, for any $\Delta<\Delta_{0}$, the compensated split-step $\theta$ method for (50) is MS-stable.

(iii) If $A<0$ and $B \geq 2\left(A\left[2 a_{1}+b_{1}^{2}+2 \lambda \mathbb{E}(\gamma)+\lambda \mathbb{E} \gamma^{2}+\right.\right.$ $\left.\left.2\left|a_{2}\right|+b_{2}^{2}+2\left|b_{1}\right|\left|b_{2}\right|\right]\right)^{1 / 2}$, then there exists a constant $\Delta_{1}$ such that, for any $\Delta<\Delta_{1}$, the compensated split-step $\theta$ method for (50) is MS-stable.

(iv) If $A>0$, then there exists a constant $\Delta_{2}$ such that, for any $\Delta<\Delta_{2}$, the compensated split-step $\theta$ method for (50) is MS-stable.

Here,

$$
\Delta_{0}=-\frac{2 a_{1}+b_{1}^{2}+2 \lambda \mathbb{E}(\gamma)+\lambda \mathbb{E}\left(|\gamma|^{2}\right)+2\left|a_{2}\right|+b_{2}^{2}+2\left|b_{1}\right|\left|b_{2}\right|}{B}
$$

$\Delta_{1}=(-B-\sqrt{\widehat{\widehat{\Delta}}}) / 2 A$ and $\Delta_{2}=(-B+\sqrt{\widehat{\widehat{\Delta}}}) / 2 A$.

Proof. From (57a), if $\theta \neq 1 / \Delta a_{1}$, we have

$$
\begin{gathered}
x_{n}^{*}=\frac{1}{1-a_{1} \theta \Delta}\left[\left(1+(1-\theta) a_{1} \Delta+\lambda \gamma_{N\left(t_{n}\right)+1} \Delta\right) x_{n}\right. \\
\left.+a_{2} \theta \Delta x_{\bar{m}}+a_{2}(1-\theta) \Delta x_{m}\right] .
\end{gathered}
$$

Then, it can be obtained that

$$
\begin{aligned}
(1- & \left.a_{1} \theta \Delta\right)^{2}\left|x_{n}^{*}\right|^{2} \\
\leq & \left(1+(1-\theta) a_{1} \Delta+\lambda \gamma_{N\left(t_{n}\right)+1} \Delta\right)^{2}\left|x_{n}\right|^{2} \\
& +a_{2}^{2} \theta^{2} \Delta^{2}\left|x_{\bar{m}}\right|^{2}+a_{2}^{2}(1-\theta)^{2} \Delta^{2}\left|x_{m}\right|^{2} \\
& +\left|a_{2}\right| \theta \Delta\left(1+(1-\theta)\left|a_{1}\right| \Delta+\lambda\left|\gamma_{N\left(t_{n}\right)+1}\right| \Delta\right) \\
& \cdot\left(\left|x_{n}\right|^{2}+\left|x_{\bar{m}}\right|^{2}\right)+\left|a_{2}\right|(1-\theta) \\
& \cdot \Delta\left(1+(1-\theta)\left|a_{1}\right| \Delta+\lambda\left|\gamma_{N\left(t_{n}\right)+1}\right| \Delta\right)\left(\left|x_{n}\right|^{2}+\left|x_{m}\right|^{2}\right) \\
& +a_{2}^{2} \theta(1-\theta) \Delta^{2}\left(\left|x_{\bar{m}}\right|^{2}+\left|x_{m}\right|^{2}\right)
\end{aligned}
$$




$$
\begin{aligned}
& \leq\left[\left(1+(1-\theta) a_{1} \Delta+\lambda \gamma_{N\left(t_{n}\right)+1} \Delta\right)^{2}\right. \\
& \leq P\left(a_{1}, a_{2}, b_{1}, b_{2}, \lambda, \mathbb{E}(\gamma)\right) \mathbb{E}\left(\left|x_{n}\right|^{2}\right) \\
& \left.+\left|a_{2}\right| \Delta\left(1+(1-\theta)\left|a_{1}\right| \Delta+\lambda\left|\gamma_{N\left(t_{n}\right)+1}\right| \Delta\right)\right]\left|x_{n}\right|^{2} \\
& +Q\left(a_{1}, a_{2}, b_{1}, b_{2}, \lambda, \mathbb{E}(\gamma)\right) \mathbb{E}\left(\left|x_{\bar{m}}\right|^{2}\right) \\
& +\left[a_{2}^{2} \theta \Delta^{2}+\left|a_{2}\right| \theta \Delta\right. \\
& \left.\cdot\left(1+(1-\theta)\left|a_{1}\right| \Delta+\lambda\left|\gamma_{N\left(t_{n}\right)+1}\right| \Delta\right)\right]\left|x_{\bar{m}}\right|^{2} \\
& +\left[a_{2}^{2}(1-\theta) \Delta^{2}+\left|a_{2}\right|(1-\theta)\right. \\
& \left.\cdot \Delta\left(1+(1-\theta)\left|a_{1}\right| \Delta+\lambda\left|\gamma_{N\left(t_{n}\right)+1}\right| \Delta\right)\right]\left|x_{m}\right|^{2} \text {. } \\
& +R\left(a_{1}, a_{2}, b_{1}, b_{2}, \lambda, \mathbb{E}(\gamma)\right) \mathbb{E}\left(\left|x_{m}\right|^{2}\right) \\
& \leq\left(P\left(a_{1}, a_{2}, b_{1}, b_{2}, \lambda, \mathbb{E}(\gamma)\right)\right. \\
& +Q\left(a_{1}, a_{2}, b_{1}, b_{2}, \lambda, \mathbb{E}(\gamma)\right) \\
& \left.+R\left(a_{1}, a_{2}, b_{1}, b_{2}, \lambda, \mathbb{E}(\gamma)\right)\right) \max _{-r \leq s \leq n} \mathbb{E}\left(\left|x_{s}\right|^{2}\right),
\end{aligned}
$$

From (57b), it is true that

$$
x_{n+1}=\left(1+b_{1} \Delta W_{n}+\gamma_{N\left(t_{n}\right)+1} \Delta \bar{N}_{n}\right) x_{n}^{*}+b_{2} \Delta W_{n} x_{m} .
$$

Hence,

$$
\begin{aligned}
\left|x_{n+1}\right|^{2}= & \left(1+b_{1} \Delta W_{n}+\gamma_{N\left(t_{n}\right)+1} \Delta \bar{N}_{n}\right)^{2}\left|x_{n}^{*}\right|^{2} \\
& +b_{2}^{2}\left(\Delta W_{n}\right)^{2}\left|x_{m}\right|^{2}+2 b_{2} \Delta W_{n} \\
& \cdot\left(1+b_{1} \Delta W_{n}+\gamma_{N\left(t_{n}\right)+1} \Delta \bar{N}_{n}\right) x_{n}^{*} x_{m} \\
\leq & \left(1+b_{1}^{2}\left(\Delta W_{n}\right)^{2}+\left(\gamma_{N\left(t_{n}\right)+1}\right)^{2}\left(\Delta \bar{N}_{n}\right)^{2}\right. \\
& +2 b_{1} \Delta W_{n}+2 \gamma_{N\left(t_{n}\right)+1} \Delta \bar{N}_{n} \\
& \left.+2 b_{1} \Delta W_{n} \gamma_{N\left(t_{n}\right)+1} \Delta \bar{N}_{n}\right)\left|x_{n}^{*}\right|^{2} \\
& +b_{2}^{2}\left(\Delta W_{n}\right)^{2}\left|x_{m}\right|^{2}+\left|b_{1} b_{2}\right|\left(\Delta W_{n}\right)^{2}\left(\left|x_{n}^{*}\right|^{2}+\left|x_{m}\right|^{2}\right) \\
& +2 b_{2} \Delta W_{n}\left(1+\gamma_{N\left(t_{n}\right)+1} \Delta \bar{N}_{n}\right) x_{n}^{*} x_{m} .
\end{aligned}
$$

Therefore,

$$
\begin{gathered}
\mathbb{E}\left(\left|x_{n+1}\right|^{2}\right) \\
\leq\left(1+b_{1}^{2} \Delta+\mathbb{E}\left(\gamma^{2}\right) \lambda \Delta+\left|b_{1} b_{2}\right| \Delta\right) \mathbb{E}\left(\left|x_{n}^{*}\right|^{2}\right) \\
+\left(b_{2}^{2} \Delta+\left|b_{1} b_{2}\right| \Delta\right) \mathbb{E}\left(\left|x_{m}\right|^{2}\right) \\
=\frac{1+b_{1} \Delta+\lambda \mathbb{E}\left(\gamma^{2}\right) \Delta+\left|b_{1} b_{2}\right| \Delta}{\left(1-a_{1} \theta \Delta\right)^{2}} \\
\cdot\left(\left[\mathbb{E}\left(\left(1+(1-\theta) a_{1} \Delta+\lambda \gamma_{N\left(t_{n}\right)+1} \Delta\right)^{2}\right)\right.\right. \\
\left.+\left|a_{2}\right| \Delta\left(1+(1-\theta)\left|a_{1}\right| \Delta+\lambda \mathbb{E}(|\gamma|) \Delta\right)\right] \\
\cdot \mathbb{E}\left(\left|x_{n}\right|^{2}\right)+\left[a_{2}^{2} \theta \Delta^{2}+\left|a_{2}\right| \theta \Delta\right. \\
\left.\cdot\left(1+(1-\theta)\left|a_{1}\right| \Delta+\lambda \mathbb{E}(|\gamma|) \Delta\right)\right] \\
\cdot \mathbb{E}\left(\left|x_{\bar{m}}\right|^{2}\right)+\left[a_{2}^{2}(1-\theta) \Delta^{2}+\left|a_{2}\right|(1-\theta) \Delta\right. \\
\left.\cdot\left(1+(1-\theta)\left|a_{1}\right| \Delta+\lambda \mathbb{E}(|\gamma|) \Delta\right)\right] \\
\left.\cdot \mathbb{E}\left(\left|x_{m}\right|^{2}\right)\right)+\left(b_{2}^{2} \Delta+\left|b_{1} b_{2}\right| \Delta\right) \mathbb{E}\left(\left|x_{m}\right|^{2}\right)
\end{gathered}
$$

where

$$
\begin{gathered}
P\left(a_{1}, a_{2}, b_{1}, b_{2}, \lambda, \mathbb{E}(\gamma)\right) \\
=\frac{1+b_{1}^{2} \Delta+\lambda \mathbb{E}\left(\gamma^{2}\right) \Delta+\left|b_{1} b_{2}\right| \Delta}{\left(1-a_{1} \theta \Delta\right)^{2}} \\
\cdot\left[\mathbb{E}\left(1+(1-\theta) a_{1} \Delta+\lambda \gamma_{N\left(t_{n}\right)+1} \Delta\right)^{2}\right. \\
\left.+\left|a_{2}\right| \Delta\left(1+(1-\theta)\left|a_{1}\right| \Delta+\lambda|\mathbb{E}(\gamma)| \Delta\right)\right] ; \\
Q\left(a_{1}, a_{2}, b_{1}, b_{2}, \lambda, \mathbb{E}(\gamma)\right) \\
=\frac{1+b_{1}^{2} \Delta+\lambda \mathbb{E}\left(\gamma^{2}\right) \Delta+\left|b_{1} b_{2}\right| \Delta}{\left(1-a_{1} \theta \Delta\right)^{2}}
\end{gathered}
$$$$
\cdot\left[a_{2}^{2} \theta \Delta^{2}+\theta\left|a_{2}\right| \Delta\left(1+(1-\theta)\left|a_{1}\right| \Delta+\lambda|\mathbb{E}(\gamma)| \Delta\right)\right] ;
$$$$
R\left(a_{1}, a_{2}, b_{1}, b_{2}, \lambda, \mathbb{E}(\gamma)\right)
$$

$$
\begin{aligned}
= & \frac{1+b_{1}^{2} \Delta+\lambda \mathbb{E}\left(\gamma^{2}\right) \Delta+\left|b_{1} b_{2}\right| \Delta}{\left(1-a_{1} \theta \Delta\right)^{2}} \\
& \cdot\left[a_{2}^{2}(1-\theta) \Delta^{2}+(1-\theta)\left|a_{2}\right| \Delta\right. \\
& \left.\cdot\left(1+(1-\theta)\left|a_{1}\right| \Delta+\lambda|\mathbb{E}(\gamma)| \Delta\right)\right] \\
& +b_{2}^{2} \Delta+\left|b_{1} b_{2}\right| \Delta .
\end{aligned}
$$

Obviously, $P\left(a_{1}, a_{2}, b_{1}, b_{2}, \lambda, \mathbb{E}(\gamma)\right)+Q\left(a_{1}, a_{2}, b_{1}, b_{2}, \lambda, \mathbb{E}(\gamma)\right)+$ $R\left(a_{1}, a_{2}, b_{1}, b_{2}, \lambda, \mathbb{E}(\gamma)\right)>0$. Then, by recursive calculation, we conclude that $\lim _{n \rightarrow \infty} \mathbb{E}\left(\left|x_{n}\right|^{2}\right)=0$ if and only if

$$
\begin{aligned}
& P\left(a_{1}, a_{2}, b_{1}, b_{2}, \lambda, \mathbb{E}(\gamma)\right)+Q\left(a_{1}, a_{2}, b_{1}, b_{2}, \lambda, \mathbb{E}(\gamma)\right) \\
& +R\left(a_{1}, a_{2}, b_{1}, b_{2}, \lambda, \mathbb{E}(\gamma)\right)<1 ;
\end{aligned}
$$


that is,

$$
\begin{aligned}
& \left(( b _ { 1 } ^ { 2 } + \lambda \mathbb { E } ( \gamma ^ { 2 } ) + | b _ { 1 } b _ { 2 } | ) \left[(1-\theta)^{2} a_{1}^{2}+\lambda^{2} \mathbb{E}\left(\gamma^{2}\right)+\left|a_{2}\right|^{2}\right.\right. \\
& +2(1-\theta) a_{1} \lambda \mathbb{E}(\gamma) \\
& \left.+2\left|a_{2}\right|\left((1-\theta)\left|a_{1}\right|+\lambda \mathbb{E}(|\gamma|)\right)\right] \\
& \left.+\theta^{2} a_{1}^{2}\left(b_{2}^{2}+\left|b_{1} b_{2}\right|\right)\right) \Delta^{2} \\
& +\left((1-2 \theta) a_{1}^{2}+\lambda^{2} \mathbb{E}\left(\gamma^{2}\right)\right. \\
& +2(1-\theta) a_{1} \lambda \mathbb{E}(\gamma)+2\left|a_{2}\right|\left((1-\theta)\left|a_{1}\right|+\lambda \mathbb{E}(|\gamma|)\right) \\
& +\left|a_{2}\right|^{2}+2\left(b_{1}^{2}+\lambda \mathbb{E}\left(\gamma^{2}\right)+\left|b_{1} b_{2}\right|\right) \\
& \cdot\left((1-\theta) a_{1}+\lambda \mathbb{E}(\gamma)+2\left|a_{2}\right|\right) \\
& \left.-2 a_{1} \theta\left(b_{2}^{2}+\left|b_{1} b_{2}\right|\right)\right) \Delta \\
& +2 a_{1}+b_{1}^{2}+2 \lambda \mathbb{E}(\gamma)+\lambda \mathbb{E}\left(|\gamma|^{2}\right)+2\left|a_{2}\right| \\
& +b_{2}^{2}+2\left|b_{1}\right|\left|b_{2}\right|<0 \text {. }
\end{aligned}
$$

Let

$$
\begin{aligned}
f(\Delta)= & A \Delta^{2}+B \Delta+2 a_{1}+b_{1}^{2}+2 \lambda \mathbb{E}(\gamma)+\lambda \mathbb{E}\left(|\gamma|^{2}\right) \\
& +2\left|a_{2}\right|+b_{2}^{2}+2\left|b_{1}\right|\left|b_{2}\right| .
\end{aligned}
$$

Then, we obtain the following conclusions.

(1) If $A=0$, then (68) can be written as

$$
\begin{aligned}
f(\Delta)= & B \Delta+2 a_{1}+b_{1}^{2}+2 \lambda \mathbb{E}(\gamma)+\lambda \mathbb{E}\left(|\gamma|^{2}\right)+2\left|a_{2}\right| \\
& +b_{2}^{2}+2\left|b_{1}\right|\left|b_{2}\right| .
\end{aligned}
$$

Furthermore,

(a) if $B \leq 0$, we have $f(\Delta)<0$ for all $\Delta>0$, which means that (66) is true for all $\Delta>0$;

(b) if $B>0$, we have $f(\Delta)<0$ for any $\Delta<-\left(2 a_{1}+\right.$ $\left.b_{1}^{2}+2 \lambda \mathbb{E}(\gamma)+\lambda \mathbb{E}\left(|\gamma|^{2}\right)+2\left|a_{2}\right|+b_{2}^{2}+2\left|b_{1}\right|\left|b_{2}\right|\right) / B$. Thus inequality (66) is hold for any

$$
\Delta<-\frac{2 a_{1}+b_{1}^{2}+2 \lambda \mathbb{E}(\gamma)+\lambda \mathbb{E}\left(|\gamma|^{2}\right)+2\left|a_{2}\right|+b_{2}^{2}+2\left|b_{1}\right|\left|b_{2}\right|}{B},
$$

which implies that (66) holds for such $\Delta$.

(2) If $A<0$, then we have the following:

(a) if $B \leq 0$, we have $f(\Delta)<0$ for all $\Delta>0$, which means that (66) is true for all $\Delta>0$;

(b) if $B>0$ and $\widehat{\Delta}=B^{2}-4 A\left[2 a_{1}+b_{1}^{2}+2 \lambda \mathbb{E}(\gamma)+\right.$ $\left.\lambda \mathbb{E}\left(|\gamma|^{2}\right)+2\left|a_{2}\right|+b_{2}^{2}+2\left|b_{1}\right|\left|b_{2}\right|\right]<0$, we have $f(\Delta)<0$ for all $\Delta>0$, which means that (66) is true for all $\Delta>0$; (c) if $B>0$ and $\widehat{\Delta}=B^{2}-4 A\left[2 a_{1}+b_{1}^{2}+2 \lambda \mathbb{E}(\gamma)+\right.$ $\left.\lambda \mathbb{E}\left(|\gamma|^{2}\right)+2\left|a_{2}\right|+b_{2}^{2}+2\left|b_{1}\right|\left|b_{2}\right|\right] \geq 0$, we have $f(\Delta)<0$ for any $\Delta<(-B-\sqrt{\widehat{\Delta}}) / 2 A$.

(3) If $A>0$, we have

$$
\begin{aligned}
\widehat{\Delta}=B^{2}-4 A[ & 2 a_{1}+b_{1}^{2}+2 \lambda \mathbb{E}(\gamma)+\lambda \mathbb{E}\left(|\gamma|^{2}\right) \\
& \left.+2\left|a_{2}\right|+b_{2}^{2}+2\left|b_{1}\right|\left|b_{2}\right|\right]>0 .
\end{aligned}
$$

Therefore, we have $f(\Delta)<0$ for any $\Delta<(-B+$ $\sqrt{\widehat{\Delta}}) / 2 A$.

The above results show that the compensated split-step $\theta$ method is of the mean-square stability for the test equations. Furthermore, it is obtained that when the parameters $a_{1}, a_{2}$, $b_{1}, b_{2}$, and $\lambda$ make the problem stable, there exists $\theta$ such that the method is stable for any time step size $\Delta$, which implies that the proposed numerical method is of the A-stable property.

\section{Conclusions}

In this paper, $A$ compensated split-step $\theta$ method is formulated to numerically solve SDEVDRJMs. It is proved that the numerical solutions converge in mean-square to their analytical solutions with such a rate that is arbitrarily close to $1 / 2$. Furthermore, the stability of the compensated splitstep $\theta$ method is investigated via a test equation and the results show that the proposed numerical method is of not only mean-square stability but also the A-stable property at least for the test equation. The results demonstrate that the proposed compensated split-step $\theta$ method is of satisfactory performance for numerically solving SDEVDRJMs.

\section{Conflict of Interests}

The authors declare that there is no conflict of interests regarding the publication of this paper.

\section{Acknowledgments}

The authors thank the anonymous referee and the editors for their careful reading of this paper and for their valuable comments. This research is funded by the National Science Foundation of China (no. 11201365).

\section{References}

[1] R. Cont and P. Taknov, Financial Modelling with Jump Process, Champman \& Hall, Boca Raton, Fla, USA, 2004.

[2] F. B. Hanson, Applied Stochastic Processes and Control for Jump-Diffusions: Modeling, Analysis and Computation, SIAM, Chicago, Ill, USA, 2007.

[3] D. J. Higham and P. E. Kloeden, "Numerical methods for nonlinear stochastic differential equations with jumps," Numerische Mathematik, vol. 101, no. 1, pp. 101-119, 2005. 
[4] D. J. Higham and P. E. Kloeden, "Convergence and stability of implicit methods for jump-diffusion systems," International Journal of Numerical Analysis and Modeling, vol. 3, no. 2, pp. 125-140, 2006.

[5] D. J. Higham and P. E. Kloeden, "Strong convergence rates for backward Euler on a class of nonlinear jump-diffusion problems," Journal of Computational and Applied Mathematics, vol. 205, no. 2, pp. 949-956, 2007.

[6] L.-S. Wang, C. Mei, and H. Xue, "The semi-implicit Euler method for stochastic differential delay equations with jumps," Applied Mathematics and Computation, vol. 192, no. 2, pp. 567578, 2007.

[7] F. Jiang, Y. Shen, and L. Liu, "Taylor approximation of the solutions of stochastic differential delay equations with Poisson jump," Communications in Nonlinear Science and Numerical Simulation, vol. 16, no. 2, pp. 798-804, 2011.

[8] J. H. Bao, X. R. Mao, and C. G. Yuan, "Convergence rate of numerical solutions to SFDEs with jumps," Journal of Computational and Applied Mathematics, vol. 236, no. 2, pp. 119-131, 2011.

[9] N. Jacob, Y. T. Wang, and C. G. Yuan, "Numerical solutions of stochastic differential delay equations with jumps," Stochastic Analysis and Applications, vol. 27, no. 4, pp. 825-853, 2009.

[10] L. Ronghua, M. Hongbing, and D. Yonghong, "Convergence of numerical solutions to stochastic delay differential equations with jumps," Applied Mathematics and Computation, vol. 172, no. 1, pp. 584-602, 2006.

[11] M. Wei, "Convergence of numerical solutions for variable delay differential equations driven by Poisson random jump measure," Applied Mathematics and Computation, vol. 212, no. 2, pp. 409-417, 2009.

[12] G. D. Chalmers and D. J. Higham, "Convergence and stability analysis for implicit simulations of stochastic differential equations with random jump magnitudes," Discrete and Continuous Dynamical Systems Series B, vol. 9, no. 1, pp. 47-64, 2008.

[13] F. B. Hanson and J. J. Westman, "Optimal consumption and portfolio control for jump-diffusion stock process with lognormal jumps," in Proceedings of the 2002 American Control Conference, vol. 5, pp. 4256-4261, Piscataway, NJ, USA, May 2002.

[14] F. Hanson and G. Yan, "Option pricing for a stochastic-volatility jump-d iffusion model with log-uniform jump amplitudes," in Proceedings of the American Control Conference, vol. 2006, pp. 1-7, Evanston, Ill, USA, June 2006.

[15] A. Svishchuk and A. Kalemanova, "The stochastic stability of interest rates with jump changes," Theory of Probability and Mathematical Statistics, vol. 61, pp. 161-172, 2000.

[16] Z. Zhu and F. B. Hanson, "A Monte-Carlo option-pricing algorithm for log-uniform jump-diffusion model," in Proceedings of the 44th IEEE Conference on Decision and Control, and the European Control Conference (CDC-ECC '05), pp. 5221-5226, Seville, Spain, December 2005.

[17] F. Jiang, Y. Shen, and L. Liu, "Numerical methods for a class of jump-diffusion systems with random magnitudes," Communications in Nonlinear Science and Numerical Simulation, vol. 16, no. 7, pp. 2720-2729, 2011.

[18] W. Mao, "Convergence analysis of semi-implicit Euler methods for solving stochastic equations with variable delays and random jump magnitudes," Journal of Computational and Applied Mathematics, vol. 235, no. 8, pp. 2569-2580, 2011.
[19] S. Mohamad and K. Gopalsamy, "Continuous and discrete Halanay-type inequalities," Bulletin of the Australian Mathematical Society, vol. 61, no. 3, pp. 371-385, 2000. 


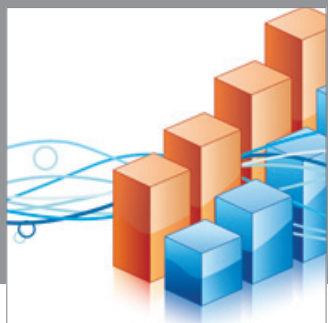

Advances in

Operations Research

mansans

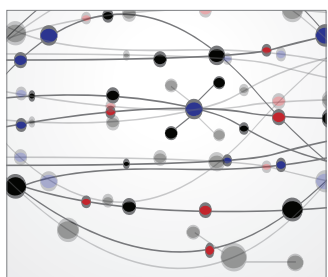

The Scientific World Journal
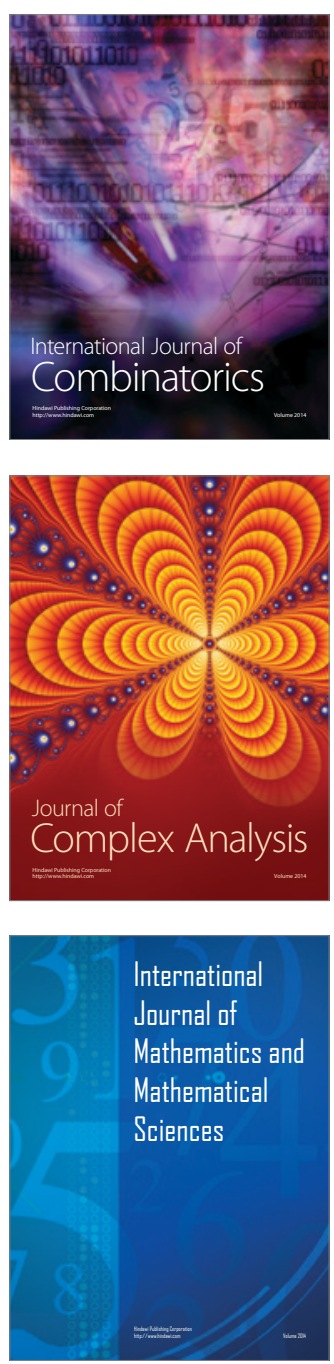
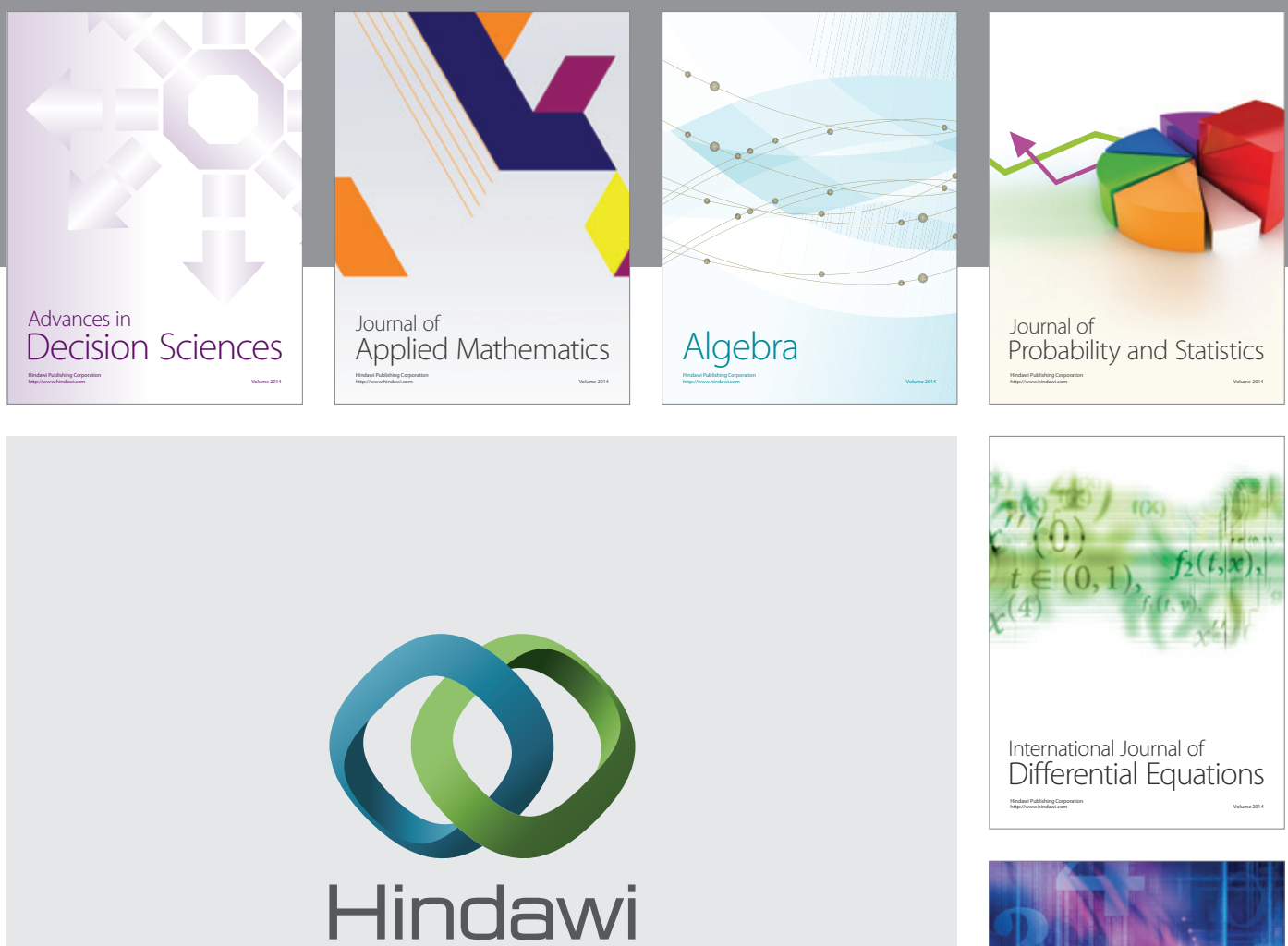

Submit your manuscripts at http://www.hindawi.com
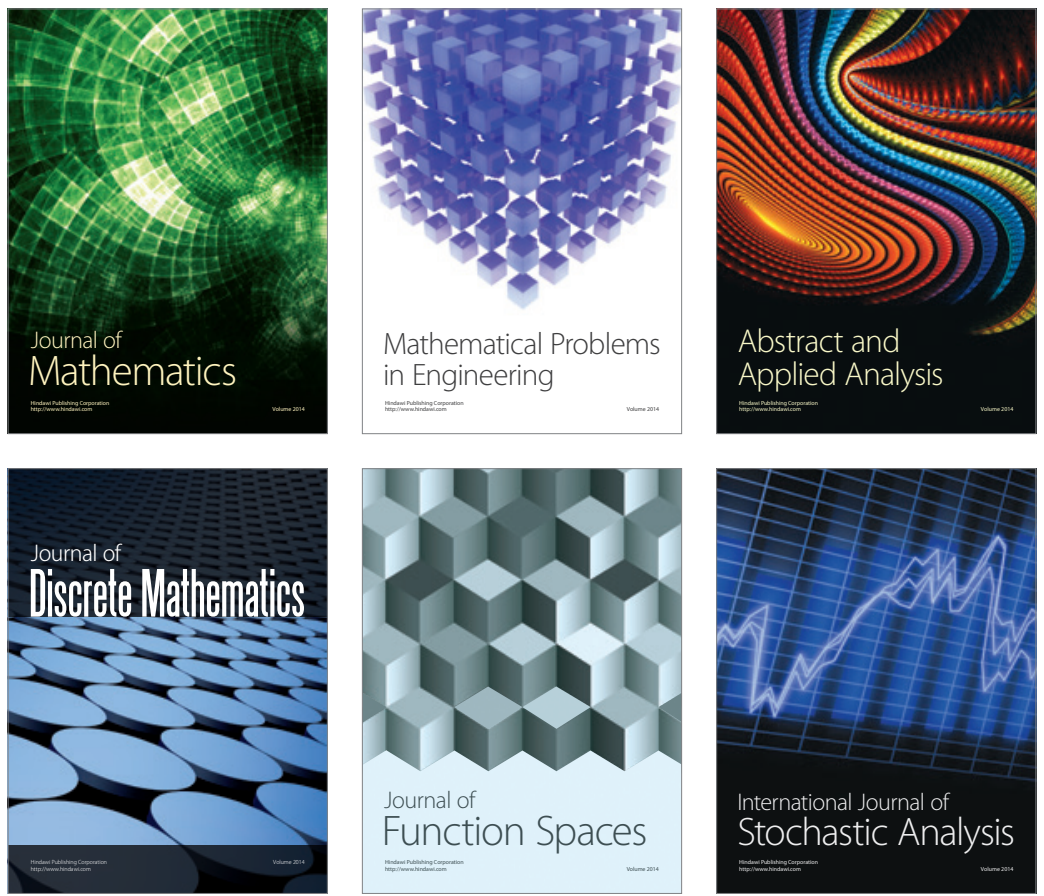

Journal of

Function Spaces

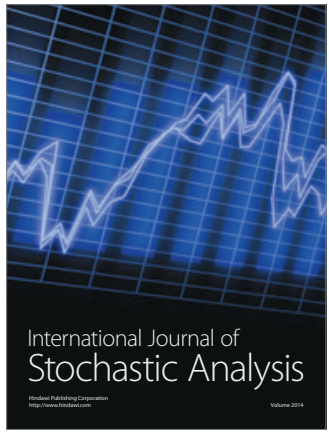

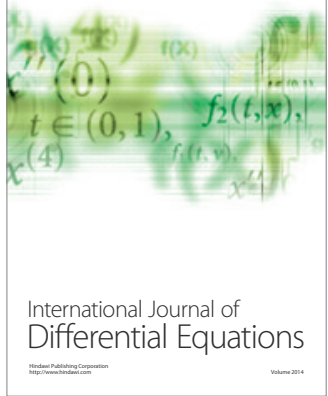
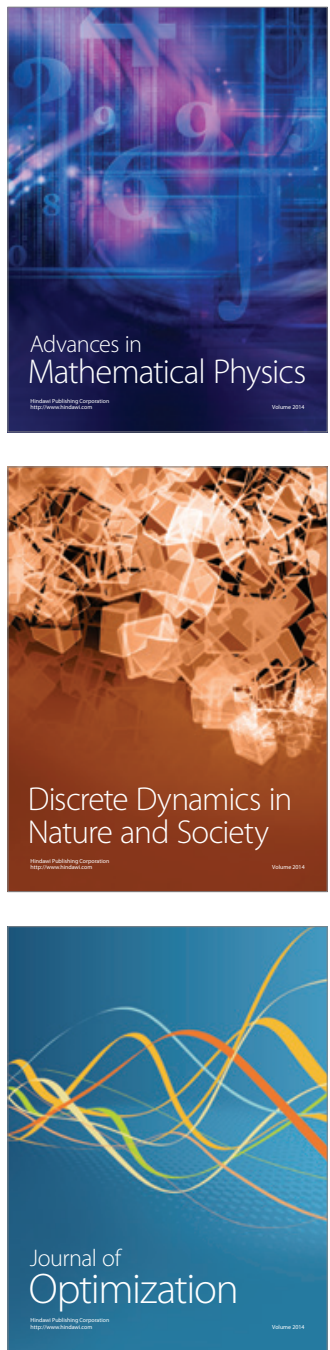